\title{
Impact of perioperative chemotherapy on survival in patients with advanced primary urethral cancer: results of the international collaboration on primary urethral carcinoma
}

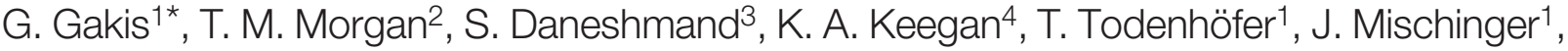 \\ T. Schubert ${ }^{1}$, H. B. Zaid ${ }^{4}$, J. Hrbacek ${ }^{5}$, B. Ali-El-Dein ${ }^{6}$, R. H. Clayman7, S. Galland ${ }^{7}$, K. Olugbade², \\ M. Rink ${ }^{8}$, H.-M. Fritsche ${ }^{9}$, M. Burger ${ }^{9}$, S. S. Chang ${ }^{4}$, M. Babjuk ${ }^{5}$, G. N. Thalmann ${ }^{10}$, A. Stenzl ${ }^{1}$ \& \\ J. A. Efstathiou ${ }^{7}$ \\ ${ }^{1}$ Department of Urology, University of Tuebingen, Tuebingen, Germany; ${ }^{2}$ Department of Urology, University of Michigan, Ann Arbor, USA; ${ }^{3}$ Institute of Urology, USC/Norris \\ Comprehensive Cancer Center, Los Angeles; ${ }^{4}$ Department of Urologic Surgery, Vanderbilt University Medical Center, Nashville, USA; ${ }^{5} 2$ nd Medical School, Department of \\ Urology, Charles University, Prague, Czech Republic; ${ }^{6}$ Urology and Nephrology Center, Mansoura Clinic, Mansoura, Egypt; ${ }^{7}$ Department of Radiooncology, Massachusetts \\ General Hospital, Harvard Medical School, Boston, USA; ${ }^{8}$ Department of Urology, University Medical Center Hamburg-Eppendorf, Hamburg; ${ }^{9}$ Department of Urology, \\ University Hospital Regensburg, Regensburg, Germany; ${ }^{10}$ Department of Urology, University Hospital Bern, Bern, Switzerland
}

Received 12 October 2014; revised 20 January 2015 and 3 April 2015; accepted 6 May 2015

Background: To investigate the impact of perioperative chemo(radio)therapy in advanced primary urethral carcinoma (PUC).

Patients and methods: A series of 124 patients (86 men, 38 women) were diagnosed with and underwent surgery for PUC in 10 referral centers between 1993 and 2012. Kaplan-Meier analysis with log-rank testing was used to investigate the impact of perioperative chemo(radio)therapy on overall survival (OS). The median follow-up was 21 months (mean: 32 months; interquartile range: 5-48).

Results: Neoadjuvant chemotherapy (NAC), neoadjuvant chemoradiotherapy (N-CRT) plus adjuvant chemotherapy $(\mathrm{ACH})$, and $\mathrm{ACH}$ was delivered in $12(31 \%), 6(15 \%)$ and 21 (54\%) of these patients, respectively. Receipt of NAC/N-CRT was associated with clinically node-positive disease $(\mathrm{cN}+; P=0.033)$ and lower utilization of cystectomy at surgery $(P=0.015)$. The objective response rate to NAC and N-CRT was $25 \%$ and $33 \%$, respectively. The 3 -year OS for patients with objective response to neoadjuvant treatment (complete/partial response) was $100 \%$ and $58.3 \%$ for those with stable or progressive disease $(P=0.30)$. Of the 26 patients staged $\geq c T 3$ and/or $\mathrm{cN}+$ disease, $16(62 \%)$ received perioperative chemo(radio)therapy and 10 upfront surgery without perioperative chemotherapy (38\%). The 3-year OS for this locally advanced subset of patients ( $\geq \mathrm{CT} 3$ and/or $\mathrm{CN}+$ ) who received NAC $(N=5), \mathrm{N}-\mathrm{CRT}(\mathrm{N}=3)$, surgery-only $(N=10)$ and surgery plus ACH ( $N=8)$ was $100 \%, 100 \%, 50 \%$ and $20 \%$, respectively $(P=0.016)$. Among these 26 patients, receipt of neoadjuvant treatment was significantly associated with improved 3 -year relapse-free survival (RFS) $(P=0.022)$ and OS $(P=0.022)$. Proximal tumor location correlated with inferior 3 -year RFS and OS $(P=0.056 / 0.005)$.

Conclusion: In this series, patients who received NAC/N-CRT for CT3 and/or CN+ PUC appeared to demonstrate improved survival compared with those who underwent upfront surgery with or without $\mathrm{ACH}$.

Key words: primary urethral carcinoma, adjuvant, neoadjuvant, chemotherapy, chemoradiotherapy

\section{introduction}

Primary urethral carcinoma (PUC) is an uncommon but potentially lethal genitourinary malignancy that meets the definition

${ }^{*}$ Correspondence to: Dr Georgios Gakis, Department of Urology, Eberhard-Karls University, University Hospital Tübingen, Hoppe-Seyler Strasse 3, D-72076 Tuebingen, Germany. Tel: +49-7071-2985092; Fax: +49-7071-295092; E-mail: georgios.gakis@ googlemail.com of a 'rare cancer,' accounting for well under $1 \%$ of all malignancies. The estimated annual incidence of PUC is 650 new cases in Europe (age-standardized ratio of 1.6/million in men and 0.6/ million in women), and based on an analysis of the Surveillance, Epidemiology and End Results registry, the age-standardized rate was reported to be three times higher in the United States (4.3/million in men and $1.5 /$ million in women) $[1,2]$. 
Prognosis of patients with PUC mainly depends on pathologic tumor and nodal stage, with distal urethral tumors exhibiting significantly improved survival rates compared with proximal tumors [3]. While surgery-only series have reported 5-year overall survival (OS) rates in advanced PUC of only $\sim 40 \%$ [4], recent retrospective studies have emphasized that modern platinumbased polychemotherapeutic regimens can be effective in prolonging survival even in lymph node-positive disease [5]. Yet, these reports have also supported the critical role of consolidative surgery after chemotherapy for achieving long-term survival in patients with locally advanced urethral cancer [5]. Therefore, optimizing treatment of advanced urethral cancer has recently become the focus of international health care authorities aiming at improving oncological efficacy and quality of life of patients with this disease [3].

Given the rarity of this cancer, there remain critical gaps in our understanding of the optimal management of patients with PUC. In particular, there are no reports we are aware of addressing the timing of perioperative chemotherapy in patients with clinically advanced PUC. In order to evaluate this clinical need, we have assembled a multi-institutional collaborative with the aim of determining the impact of neoadjuvant and adjuvant treatment in patients undergoing surgery for PUC.

\section{patients and methods}

\section{patient cohort}

In this Institutional-Review Board approved retrospective observational multicenter analysis, we reviewed the clinical and pathologic records of a total of 124 consecutive patients ( 86 men, 38 women) obtained from 10 prospectively maintained databases who underwent surgery for PUC at 10 academic centers between 1993 and 2012. Patients with evidence of distant metastatic disease on cross-sectional imaging before primary treatment were excluded from analysis.

\section{perioperative treatment and assessment of response}

In order to understand the timing of perioperative chemotherapy, this study assessed the subset of patients receiving neoadjuvant chemotherapy (NAC), neoadjuvant chemoradiotherapy (N-CRT) and adjuvant chemotherapy (ACH) around the time of surgical resection. Assessment of response to neoadjuvant treatment was based on endoscopic and cross-sectional imaging findings. The number of treatment cycles and the time interval between perioperative chemo (radio)therapy and surgery was recorded. Objective response to neoadjuvant chemo(radio)therapy was defined as partial (PR) or complete response (CR) [5]. $\mathrm{ACH}$ was administered to the discretion of the treating physician based on pathological risk factors in specimens. For those receiving N-CRT, a dose of 40$45 \mathrm{~Gy}$ was delivered to the pelvic region by external beam radiotherapy with an additional boost to the primary tumor of 20-24 Gy delivered either by intensitymodulated radiation therapy or by brachytherapy. To better understand the role of surgery in advanced stages, outcomes of patients who underwent surgeryonly for $\geq \mathrm{cT} 3$ and/or $\mathrm{cN}+$ disease $(N=10)$ were additionally evaluated.

\section{surgery}

The modality of surgical treatment included transurethral resection, partial/ total urethrectomy and radical cystectomy with urethrectomy and urinary diversion. Bilateral regional lymph node dissection (LND) was carried out at the discretion of the treating surgeon based on intraoperative findings and preoperative cross-sectional imaging. The level of LND was based on the location of the primary tumor and typically included the inguinal lymph nodes, external and internal iliac, obturator and common iliac lymph nodes.

\section{clinical and histologic assessment}

The following clinical and pathologic parameters were assessed: age at surgery, gender, clinical and pathologic tumor stage, clinical and pathologic lymph node tumor involvement, underlying histology, tumor grade, tumor location (proximal versus distal), preoperative serum creatinine level, modality of surgery for primary treatment and modality of treatment of recurrence. In men, proximal tumor location was defined as tumors located in the prostatic, membranous or bulbar urethra and anteriorly when located in the penile urethra and fossa navicularis. In women, proximal tumor location was defined as tumors located in the proximal two thirds of the urethra and anteriorly when located in the distal third [6].

The histologic assessment was carried out at the center-specific pathology department and was based on the WHO grading system and tumor-nodemetastasis classification as approved by the AJCC [7]. The pathologic macro- and microscopic evaluation of specimens included cross-sectioning of the entire specimen with immunohistochemical staining to identify the presence of urothelial, squamous cell and adenocarcinoma or rarer entities [8]. Lymphovascular invasion was defined as the presence of malignant cells within an endothelial cell line [9].

\section{follow-up}

Electronic hospital charts and physician records were reviewed to determine clinical outcomes. Patients generally were seen postoperatively at least every 3-4 months for the first year, semiannually for the second and third years, and annually thereafter. Follow-up examinations included cross-sectional imaging with computed tomography or magnetic resonance imaging. In addition to physical examination with laboratory testing, i.v. pyelography, cystoscopy, urine cytology, urethral washings and bone scintigraphy were carried out if indicated. Kaplan-Meier analysis with log-rank testing was used to investigate the impact of perioperative chemotherapy on relapse-free survival (RFS) and OS. Relapse was defined as either local recurrence in the surgical field, intraurethrally or in distant organs. For determining RFS, clinical outcomes were measured from the date of surgery to the date of first documented relapse or last follow-up visit when the patients had not experienced relapse. For OS, the date of death was determined by death certificates or hospital charts or the last follow-up visit when patients were still alive [10].

\section{statistical analysis}

For univariable analysis, $\chi^{2}$ and Fisher's exact tests were used for nominal data and Student's $t$-test for scaled data. Kaplan-Meier plots were used to estimate RFS and OS using log-rank testing.

$P$ values are two-sided and $P<0.05$ was considered significant. Statistical analysis was carried out using JMP ${ }^{\circledR}$ 11.0. Values are given as mean \pm SEM for normally distributed or as median (range) for non-normally distributed variables.

\section{results}

In the total cohort $(N=124)$, the median follow-up was 21 months [mean: 32 months; interquartile range (IQR): 5-48, total range: 3-200]. The median age at surgery was 66 years (IQR: 58-76). Of the 124 patients, stage cT3 and/or cN+ was present in $26(21 \%)$. Of these 26 patients, 16 (62\%) received perioperative chemotherapy and $10(38 \%)$ surgery without perioperative chemotherapy. A flowchart describing the selection process of the included patients is provided in supplementary File S1, available at Annals of Oncology online. 
Perioperative chemotherapy was administered in 39 patients (31\%). Of these 39 patients, 12 (31\%) received NAC, 6 (15\%) $\mathrm{N}$-CRT plus ACH and 21 (54\%) ACH. The different chemotherapeutic regimens administered for perioperative chemotherapy are listed in Table 1 . The median number (total range) of cycles of NAC, N-CRT and ACH was 3 (2-6), 3 (1-4) and 4 (2-6), respectively $(P=0.52)$. The median time interval (total range, in days) between surgery and NAC, N-CRT and ACH only was 36 (23-102), 80 (53-113) and 59 (28-600), $P=0.48$, respectively. The objective response rate to NAC and N-CRT (defined as CR or PR) was $25 \%$ and $33 \%$, respectively (see Table 1 ). The 3 -year OS for patients with objective response to neoadjuvant treatment (CR, PR) was $100 \%$ and $58.3 \%$ for those with stable disease or progressive disease $(P=0.30)$.

Among patients treated with perioperative chemotherapy, receipt of neoadjuvant chemo(radio)therapy was significantly associated with clinically node-positive tumor stage $(\mathrm{cN}+$; $P=0.046)$ and a lower utilization rate of radical cystectomy at surgery $(P=0.015)$. No significant associations were found

Table 1. Chemotherapeutic regimens and response to neoadjuvant treatment in the 39 patients undergoing perioperative treatment of primary urethral carcinoma

\begin{tabular}{|c|c|c|c|}
\hline & NAC & $\begin{array}{l}\mathrm{N}-\mathrm{CRT}+ \\
\mathrm{ACH}\end{array}$ & $\mathrm{ACH}$ \\
\hline Number of patients (\%) & $12(31)$ & $6(15)$ & $21(54)$ \\
\hline \multicolumn{4}{|l|}{ Mitomycin C/5-FU-based } \\
\hline Mitomycin C/5-FU & 0 & $1(\mathrm{SD})$ & 0 \\
\hline 5-FU only & 0 & 0 & 1 \\
\hline \multicolumn{4}{|l|}{ Cisplatinum-based } \\
\hline Cisplatin/gemcitabine & $2(\mathrm{CR}, \mathrm{PD})$ & $2(\mathrm{CR}, \mathrm{PR})$ & 4 \\
\hline Cisplatin/gemcitabine/paclitaxel & $2(\mathrm{PR}, \mathrm{SD})$ & $1(\mathrm{PD})$ & 1 \\
\hline Dose-dense MVAC & $1(\mathrm{PR})$ & 0 & 0 \\
\hline MVAC & $1(\mathrm{PD})$ & 0 & 0 \\
\hline Cisplatin/paclitaxel/ifosfamide & $1(\mathrm{PD})$ & 0 & 1 \\
\hline Cisplatin/paclitaxel/5-FU & $1(\mathrm{SD})$ & 0 & 0 \\
\hline Cisplatin/paclitaxel & $1(\mathrm{PD})$ & 0 & 5 \\
\hline Cisplatin only & $1(\mathrm{PD})$ & $1(\mathrm{PD})$ & 1 \\
\hline \multicolumn{4}{|l|}{ Carboplatinum-based } \\
\hline $\begin{array}{l}\text { Paclitaxel/carboplatin/ } \\
\text { gemcitabine }\end{array}$ & $1(\mathrm{PD})$ & 0 & 0 \\
\hline Paclitaxel/carboplatin & 0 & 0 & 1 \\
\hline $\begin{array}{l}\text { Carboplatin/gemcitabine/ } \\
\text { abraxane }\end{array}$ & $1(\mathrm{SD})$ & 0 & 0 \\
\hline Carboplatin/paclitaxel/ifosfamide & 0 & 0 & 2 \\
\hline Carboplatin/gemcitabine & 0 & 0 & 1 \\
\hline \multicolumn{4}{|l|}{ Gemcitabine-based } \\
\hline Gemcitabine/paclitaxel & 0 & $1(\mathrm{PD})$ & 1 \\
\hline Taxol/gemcitabine & 0 & 0 & 1 \\
\hline \multicolumn{4}{|l|}{ Other regimens } \\
\hline Carmustin/IL-2 & 0 & 0 & 1 \\
\hline Dacarbazine & 0 & 0 & 1 \\
\hline
\end{tabular}

5-FU, 5-fluorouracil; ACH, adjuvant chemotherapy; CR, complete response; IL-2, interleukin-2; MVAC, methotrexat/cisplatin/doxorubicin/vinblastin; NAC, neoadjuvant chemotherapy; N-CRT, neoadjuvant chemoradiotherapy; $\mathrm{PR}$, partial response; $\mathrm{PD}$, progressive disease; $\mathrm{SD}$, stable disease. between the timing of perioperative chemotherapy and age, gender, clinical and pathologic tumor stage, tumor location (proximal versus distal), underlying histology, tumor grade, preoperative serum creatinine level and modality of treatment of relapse (see Table 2).

Relapse occurred in 23 of the 39 patients (59\%) with a corresponding 3-year RFS of 54\%. In univariable analysis, RFS was only significantly associated with pathologically advanced tumor stage ( $\geq$ pT3, $P=0.034)$. There were a total of $12(30.8 \%)$ deaths during the study period, and OS was similarly only associated with pathologically advanced tumor stage $(P=0.030)$. No significant associations were found between RFS/OS and modality and number of chemotherapy cycles administered, age, gender, pathological nodal involvement, tumor location, tumor grade, clinical tumor and nodal stage, histological subtype and modality of treatment of relapse (see Table 3 ).

In the 39 patients receiving perioperative chemo(radio) therapy, the type of chemotherapy (cisplatin- versus noncisplatin-based; $57.2 \%$ versus $57.8 \% ; P=0.63)$ and the number of administered chemotherapeutic cycles ( $\geq 4$ versus $<4 ; 61.7 \%$ versus $48.9 \%, P=0.34$ ) were not associated with improved 3-year OS.

Among the 16 patients treated with perioperative chemotherapy for $\mathrm{cT} 3$ and/or $\mathrm{cN}+$ disease, the 3-year OS was $61 \%$, respectively. The 3-year OS for patients with $\geq \mathrm{cT} 3$ and/or $\mathrm{cN}+$ disease who received NAC $(N=5), \mathrm{N}$-CRT $(N=3)$, surgery-only $(N=10)$ or surgery plus $\mathrm{ACH}(N=8)$ was $100 \%, 100 \%, 50 \%$ and $20 \%$, respectively $(P=0.016)$. Among these 26 patients, receipt of neoadjuvant treatment was significantly associated with improved 3 -year RFS $(P=0.022)$ and OS $(P=0.022)$. Proximal tumor location correlated with inferior 3-year RFS and OS $(P=0.056 / 0.005)$. No significant differences were found for other parameter listed in Table 3.

\section{discussion}

The optimal timing of perioperative chemotherapy in patients with advanced PUC is unknown. Since PUC is a rare tumor entity, we set up a collaborative database and accrued a total of 124 cases to inform the role of perioperative chemotherapy. Of these 124 patients, 39 received perioperative chemotherapy (31\%).

As would be expected, delivery of NAC was associated with clinically node-positive disease. This finding suggests that the decision making for NAC in this cohort was based on evidence of nodal involvement at cross-sectional imaging. In our cohort, patients with advanced clinical stage $(\geq \mathrm{cT} 3$ and/or $\mathrm{cN}+$ ) were more likely to undergo perioperative chemotherapy before surgery (62\% versus $38 \%$ ). Interestingly, patients with clinically advanced tumor stage and/or node-positive disease exhibited improved RFS and OS when treated in the neoadjuvant setting with either NAC or N-CRT compared with patients treated only with surgery or surgery plus ACH. Among the 39 patients, RFS and OS were only associated with pathologically advanced tumor stage at surgery but not with modality and duration (cycles) of perioperative chemotherapy. These findings underline the prognostic impact of pathologic tumor extent after completion of perioperative chemotherapy. Although the objective response rate of neoadjuvant treatment was relatively low, administration of neoadjuvant treatment was associated with 
Table 2. Univariable Pearson's $\chi^{2}$ Fisher's exact test for clinical and pathologic parameters in the 39 patients receiving perioperative chemotherapy

\begin{tabular}{|c|c|c|c|c|}
\hline & NAC & $\begin{array}{l}\mathrm{N}-\mathrm{CRT}+ \\
\mathrm{ACH}\end{array}$ & $\mathrm{ACH}$ & $P$ value \\
\hline Number of patients (\%) & $12(31)$ & $6(15)$ & $21(54)$ & \\
\hline \multicolumn{5}{|l|}{ Follow-up time } \\
\hline Median & 12 & 30 & 36 & \multirow[t]{3}{*}{0.23} \\
\hline Mean & 19 & 30 & 24 & \\
\hline IQR & $3-50$ & $3-60$ & $4-60$ & \\
\hline \multicolumn{5}{|l|}{ Gender } \\
\hline Male & $9(75.0)$ & $3(50.0)$ & $14(66.7)$ & \multirow[t]{2}{*}{0.57} \\
\hline Female & $3(25.0)$ & $3(50.0)$ & $7(33.3)$ & \\
\hline \multicolumn{5}{|l|}{ Age } \\
\hline Median & 61 & 59 & 66 & \multirow[t]{2}{*}{0.95} \\
\hline IQR & $56-74$ & $49-66$ & $62-71$ & \\
\hline \multicolumn{5}{|l|}{ cT stage } \\
\hline cTX & $0(0)$ & $0(0)$ & $0(0)$ & \multirow[t]{9}{*}{0.10} \\
\hline $\mathrm{cTa}$ & $1(8.3)$ & $0(0)$ & $2(9.5)$ & \\
\hline cTis & $0(0)$ & $0(0)$ & $0(0)$ & \\
\hline $\mathrm{cT} 1$ & $2(16.7)$ & $1(16.7)$ & $6(28.6)$ & \\
\hline cT2 & $5(41.7)$ & $2(33.3)$ & $5(23.8)$ & \\
\hline cT3 & $2(16.7)$ & $2(33.3)$ & $3(14.3)$ & \\
\hline cT4 & $2(16.7)$ & $1(16.7)$ & $5(23.8)$ & \\
\hline cTa-T2 & $8(66.7)$ & $3(50.0)$ & $13(61.9)$ & \\
\hline cT3-T4 & $4(33.3)$ & $3(50.0)$ & $8(38.1)$ & \\
\hline \multicolumn{5}{|l|}{ pT stage } \\
\hline pT0 & $0(0)$ & $0(0)$ & $0(0)$ & \multirow[t]{9}{*}{0.58} \\
\hline $\mathrm{pTa}$ & $0(0)$ & $0(0)$ & $0(0)$ & \\
\hline $\mathrm{p}$ Tis & $1(8.3)$ & $0(0)$ & $1(4.8)$ & \\
\hline pT1 & $0(0)$ & $0(0)$ & $5(23.8)$ & \\
\hline pT2 & $5(41.7)$ & $2(33.3)$ & $6(28.6)$ & \\
\hline pT3 & $5(41.7)$ & $1(16.7)$ & $2(9.5)$ & \\
\hline pT4 & $1(8.3)$ & $3(50.0)$ & $7(33.3)$ & \\
\hline pTa-T2 & $6(50.0)$ & $2(33.3)$ & $12(57.1)$ & \\
\hline pT3-T4 & $6(50.0)$ & $4(66.7)$ & $9(42.9)$ & \\
\hline \multicolumn{5}{|l|}{$\mathrm{cN}$ stage } \\
\hline $\mathrm{cNX}$ & $3(25.0)$ & $2(33.3)$ & $0(0)$ & \multirow[t]{3}{*}{0.046} \\
\hline cNo & $5(41.7)$ & $2(33.3)$ & $17(81.0)$ & \\
\hline $\mathrm{cN}+$ & $4(33.3)$ & $2(33.3)$ & $4(19.0)$ & \\
\hline \multicolumn{5}{|l|}{ pN stage } \\
\hline $\mathrm{pNX}$ & $6(50.0)$ & $2(33.3)$ & $7(33.3)$ & \multirow[t]{3}{*}{0.07} \\
\hline pNo & $3(25.0)$ & $1(16.7)$ & $12(57.1)$ & \\
\hline pN1-2 & $3(25.0)$ & $3(50.0)$ & $2(9.5)$ & \\
\hline \multicolumn{5}{|c|}{ Tumor grade at primary diagnosis } \\
\hline G1 & $0(0)$ & $1(16.7)$ & $0(0)$ & \multirow[t]{3}{*}{0.18} \\
\hline G2 & $3(25.0)$ & $0(0)$ & $3(14.3)$ & \\
\hline G3 & $9(75.0)$ & $5(83.3)$ & $18(85.7)$ & \\
\hline \multicolumn{5}{|l|}{ Histology } \\
\hline UC & $4(33.3)$ & $1(16.7)$ & $12(57.1)$ & \multirow[t]{5}{*}{0.23} \\
\hline SCC & $5(41.7)$ & $3(50.0)$ & $3(14.3)$ & \\
\hline $\mathrm{AC}$ & $2(16.7)$ & $2(33.3)$ & $2(9.5)$ & \\
\hline Mixed & $1(8.3)$ & $0(0)$ & $2(9.5)$ & \\
\hline Unclassified & $0(0)$ & $0(0)$ & $2(9.5)$ & \\
\hline \multicolumn{5}{|l|}{ Tumor location } \\
\hline Proximal & $7(58.3)$ & $3(50.0)$ & $14(66.7)$ & \multirow[t]{2}{*}{0.44} \\
\hline Distal & $5(41.7)$ & $3(50.0)$ & $7(33.3)$ & \\
\hline
\end{tabular}

Table 2. Continued

\begin{tabular}{|c|c|c|c|c|}
\hline & NAC & $\begin{array}{l}\mathrm{N}-\mathrm{CRT}+ \\
\mathrm{ACH}\end{array}$ & $\mathrm{ACH}$ & $P$ value \\
\hline \multicolumn{5}{|c|}{ Preoperative serum creatinine level (mg/dl) } \\
\hline Median & 0.9 & 0.9 & 1.0 & \multirow[t]{3}{*}{0.70} \\
\hline Mean & 1.0 & 0.9 & 1.0 & \\
\hline IQR & $0.8-1.2$ & $0.7-1.1$ & $0.7-1.2$ & \\
\hline \multicolumn{5}{|c|}{ Surgical modality of primary treatment } \\
\hline TUR only & $1(8.3)$ & $0(0)$ & $5(23.8)$ & \multirow[t]{5}{*}{0.015} \\
\hline Partial urethrectomy & $0(0)$ & $1(16.7)$ & $0(0)$ & \\
\hline Urethrectomy & $5(41.7)$ & $2(33.3)$ & $5(23.8)$ & \\
\hline $\begin{array}{l}\text { Cyst(oprostat)ectomy } \\
\text { plus urethrectomy }\end{array}$ & $3(25.0)$ & $0(0)$ & $11(52.4)$ & \\
\hline Other & $3(25.0)$ & $3(50.0)$ & $0(0)$ & \\
\hline \multicolumn{5}{|c|}{ Modality of treatment of local/urethral relapse } \\
\hline Surgery & $3(25.0)$ & $0(0)$ & $3(14.3)$ & 0.41 \\
\hline Radiotherapy & $3(25.0)$ & $0(0)$ & $3(14.3)$ & 0.63 \\
\hline Chemotherapy & $0(0)$ & $0(0)$ & $0(0)$ & - \\
\hline $\begin{array}{l}\text { Receipt of palliative } \\
\text { (systemic) } \\
\text { chemotherapy }\end{array}$ & $3(25.0)$ & $1(16.7)$ & $9(42.9)$ & 0.36 \\
\hline $\begin{array}{l}\text { AC, adenocarcinoma; } \mathrm{AC} \\
\text { range; NAC, neoadjuvant } \\
\text { diotherapy; SCC, squam } \\
\text { tion; UC, urothelial carci }\end{array}$ & $\begin{array}{l}\text { s cell car } \\
\text { ma. }\end{array}$ & $\begin{array}{l}\text { chemother } \\
\text { py; N-CRT, } \\
\text { inoma; TU }\end{array}$ & $\begin{array}{l}\text {; IQR, int } \\
\text { oadjuvant } \\
\text { transureth }\end{array}$ & $\begin{array}{l}\text { rquartile } \\
\text { hemora- } \\
\text { al resec- }\end{array}$ \\
\hline
\end{tabular}

improved 3-year RFS and OS in the subset of patients with locally advanced disease. Additionally, proximal tumor location was associated with inferior RFS and OS which is in line with prior studies $[1,2,4]$. These data suggest that patients with proximal tumor location and clinically advanced stages may benefit most from neoadjuvant treatment. Despite the fact that patients treated with neoadjuvant chemo(radio)therapy had similar rates of pathologically advanced stages and proximal tumor location at surgery compared with patients treated with adjuvant therapy, they were less likely to undergo cystectomy. Similar to bladder cancer [11], this finding hints at the possibility that neoadjuvant chemo(radio) therapy may exert a beneficial impact on the primary tumor extent and facilitate the surgical approach.

We found that OS rates in patients with clinically advanced stages ( $\geq \mathrm{cT} 3$ and/or $\mathrm{cN}+$ ) did not differ between those treated either with $\mathrm{NAC}$ or with $\mathrm{N}-\mathrm{CRT}$ plus $\mathrm{ACH}$ whereas $\mathrm{ACH}$ was associated with decreased OS. The underlying histology did not influence survival. These findings suggest that delivery of neoadjuvant chemo(radio)therapy is critical for survival in advanced stages. Owing to these findings and the low number of patients, we combined patients treated with NAC and N-CRT plus ACH into one group for final survival analysis.

Although the number of included cases is low and the present results have to be interpreted carefully, some meaningful conclusions can be drawn from these results. First, our findings suggest that the presence of clinically enlarged lymph nodes should alert clinicians of the possible presence of lymph node metastatic disease, especially in case of advanced clinical tumor stage and proximal tumor location, and should therefore be an 
Table 3. Relapse-free and overall survival for patients treated with or without perioperative chemo(radio)therapy according to clinical and pathologic tumor characteristics

\begin{tabular}{|c|c|c|c|c|c|c|c|c|}
\hline \multirow[t]{3}{*}{ Parameters } & \multicolumn{4}{|c|}{ Total cohort with perioperative chemotherapy $(N=39)$} & \multicolumn{4}{|c|}{ cT3 and/or cN+ (with or without perioperative chemotherapy) $(N=26)$} \\
\hline & \multicolumn{2}{|c|}{ RFS } & \multicolumn{2}{|l|}{ OS } & \multicolumn{2}{|c|}{ RFS } & \multicolumn{2}{|l|}{ OS } \\
\hline & $\mathrm{RR}(95 \% \mathrm{CI})$ & $P$ value & $\mathrm{RR}(95 \% \mathrm{CI})$ & $P$ value & $\mathrm{RR}(95 \% \mathrm{CI})$ & $P$ value & $\mathrm{RR}(95 \% \mathrm{CI})$ & $P$ value \\
\hline NAC/N-CRT plus ACH versus ACH only & $0.91(0.42-1.90)$ & 0.97 & $0.98(0.32-2.96)$ & 0.80 & $\begin{array}{l}0.14(0.01-0.78) \text { (for } \\
\text { NAC/N-CRT plus ACH } \\
\text { versus ACH/surgery-only) }\end{array}$ & 0.022 & $\begin{array}{l}0.10(0.01-0.71) \text { (for } \\
\text { NAC/N-CRT plus ACH } \\
\text { versus ACH/surgery-only) }\end{array}$ & 0.024 \\
\hline$\geq \mathrm{pT} 3$ versus $\leq \mathrm{pT} 2$ & $2.6(1.1-6.8)$ & 0.030 & $5.5(1.2-35.4)$ & 0.034 & $1.23(0.43-4.47)$ & 0.70 & $1.02(0.22-7.16)$ & 0.98 \\
\hline$\geq \mathrm{cT} 3$ versus $\leq \mathrm{cT} 2$ & $2.1(0.9-5.3)$ & 0.26 & $2.3(0.5-9.7)$ & 0.10 & - & - & - & - \\
\hline $\mathrm{cN}+$ versus $\mathrm{cN} 0$ & $2.5(0.8-7.2)$ & 0.25 & $2.5(0.5-11.6)$ & 0.11 & - & - & - & - \\
\hline $\mathrm{cNX}$ versus $\mathrm{cN} 0$ & $4.1(0.2-28.9)$ & 0.17 & $7.0(0.3-68.39)$ & 0.28 & - & - & - & - \\
\hline Tumor location proximal versus distal & $1.9(0.7-5.4)$ & 0.35 & $2.1(0.5-14.3)$ & 0.20 & $2.33(0.98-5.95)$ & 0.056 & $10.11(1.82-188.90)$ & 0.005 \\
\hline Tumor grade G1/G2 versus G3 & $2.3(0.7-10.0)$ & 0.21 & $3.2(0.6-59.8)$ & 0.17 & $5.97(0.57-50.61)$ & 0.13 & $4.66(0.84-86.90)$ & 0.08 \\
\hline \multicolumn{9}{|l|}{ Histology } \\
\hline UC versus SCC & $0.7(0.2-2.6)$ & 0.49 & $1.1(0.1-22.0)$ & 0.53 & $1.10(0.41-3.04)$ & 0.84 & $2.17(0.41-15.95)$ & 0.36 \\
\hline UC versus AC & $0.3(0.1-1.4)$ & 0.84 & $0.8(0.1-16.2)$ & 0.13 & $0.66(0.04-3.71)$ & 0.68 & $0.01(0-3.27)$ & 0.23 \\
\hline \multicolumn{9}{|l|}{ Gender } \\
\hline Male versus female & $0.6(0.2-1.7)$ & 0.13 & $0.3(0.1-1.4)$ & 0.32 & $1.23(0.54-2.86)$ & 0.61 & $0.54(0.11-2.05)$ & 0.36 \\
\hline \multicolumn{9}{|l|}{ Age } \\
\hline$>65$ years versus $\leq 65$ years & $1.7(0.5-6.4)$ & 0.59 & $1.6(0.3-11.4)$ & 0.38 & $1.48(0.59-3.76)$ & 0.39 & $1.23(0.29-5.22)$ & 0.77 \\
\hline \multicolumn{9}{|l|}{ Treatment of relapse } \\
\hline Surgery & - & - & $0.4(0.1-1.2)$ & 0.08 & - & - & $0.19(0.01-1.17)$ & 0.07 \\
\hline Radiotherapy & - & - & $0.3(0-1.5)$ & 0.15 & - & - & $2.28(0.31-11.76)$ & 0.31 \\
\hline Palliative (systemic) chemotherapy & - & - & $1.4(0.6-3.4)$ & 0.42 & - & - & $2.56(0.52-10.50)$ & 0.22 \\
\hline
\end{tabular}


impetus to deliver neoadjuvant chemo(radio)therapy. This finding is in line with studies reporting a high degree of concordance between clinical and pathological staging in PUC [3]. Another explanatory approach for the worse prognosis of patients with $\mathrm{ACH}$ may be that utilization of radical cystectomy with urinary diversion adversely affects performance status and postoperative renal function thereby delaying timely delivery of chemotherapy after surgery [11]. However, in our cohort, preoperative serum creatinine levels were not different between the three groups which suggests that renal function did not impact on the decision making for perioperative treatment.

Similar to our findings, a prior series reported outcomes in 44 patients treated uniformly with chemotherapy without radiotherapy for advanced primary urethral cancer with squamous cell carcinoma (SCC) and adenocarcinoma being the most prevalent histological entities. Patients were subjected to specific cisplatin-based polychemotherapeutic regimens according to the underlying histology. The overall response rate for the various regimens was reported to be much higher with $72 \%$. The median OS of the entire cohort was 32 months. Of note, patients who underwent surgery after chemotherapy had significantly improved OS compared with those who were managed with chemotherapy alone [5].

Another recent series reported that $\mathrm{CR}$ to concurrent chemoradiotherapy (consisting of two cycles of 5-fluorouracil/mitomycin C and 45-55 Gy over the span of 5 weeks) in T3 SCC of the urethra was observed in $79 \%$ of the patients. Consolidation surgery was, however, only initiated if patients never responded to their 'modified Nigro-protocol' or developed local recurrence. Despite the high primary response rate of $\sim 80 \%$, the corresponding 5 -year disease-free and OS rates were moderate with $43 \%$ and $52 \%$ [12].

In our series, we found that the 3-year OS rate in patients with $\geq \mathrm{cT} 3$ and/or $\mathrm{cN}+$ stage was relatively high at $61 \%$. Of note, all patients treated with NAC/N-CRT were alive after 5 years. Therefore, these data suggest that consolidation surgery may be of prognostic importance at the time of completion of neoadjuvant chemo(radio)therapy which may also obviate the need for aggressive salvage surgery in case of relapse. In terms of the effects of salvage treatment on OS, we found that neither surgery nor radiotherapy exerted a beneficial impact on survival. These data highlight, once more, the importance of the decision making for primary treatment in advanced PUC. In this regard, we recently reported that clinical nodal stage is a strong predictor for oncological outcomes in primary urethral cancer [13].

Our study has several limitations inherent to the retrospective, small size, and multicenter nature of the analysis, which of course is requisite given the rarity of the disease. Possible biases include absence of regional LND in approximately half of the patients and interobserver variabilities in the clinical staging and pathological assessment of specimens. We could not adjust for patient preferences, toxicities of perioperative treatment and comorbidities which may have impacted on the clinical decision making for either $\mathrm{NAC}$ or $\mathrm{ACH}$. In this regard, an important alternative explanation for these findings is that patients who were healthier (with better renal function) received neoadjuvant chemo(radio)therapy and less healthy patients did not, particularly in the setting of advanced disease. Yet, preoperative serum creatinine levels were not different between the three groups and all patients were fit enough to undergo either $\mathrm{ACH}$ after surgery or neoadjuvant treatment before surgery. In addition, all patients were treated in academic centers by multidisciplinary teams dedicated to the management of lower urinary tract cancer. Notwithstanding, owing to these limitations, the results of this multicenter pooled analysis provide new insights into the management of this rare disease and await further prospective validation.

This is, to our knowledge, the first series that demonstrates the prognostic benefit of NAC and N-CRT with $\mathrm{ACH}$ in a consecutive series of patients who underwent perioperative chemotherapy plus surgery for advanced PUC. Further studies should yield a better understanding of how perioperative chemotherapy exerts a positive effect on survival in order to selectively advocate its use in advanced PUC.

\section{conclusion}

In this series, patients who received NAC or chemoradiotherapy for locally advanced PUC ( $\geq \mathrm{cT} 3$ and/or $\mathrm{cN}+$ ) appeared to demonstrate improved survival compared with those who underwent upfront surgery with or without $\mathrm{ACH}$.

\section{disclosure}

The authors have declared no conflicts of interest.

\section{references}

1. Visser 0 , Adolfsson J, Rossi $S$ et al. The RARECARE working group: incidence and survival of rare urogenital cancers in Europe. Eur J Cancer 2011; 48: 456-464.

2. Swartz MA, Porter M, Lin DW, Weiss NS. Incidence of primary urethral carcinoma in the United States. Urology 2006; 68: 1164-1168.

3. Gakis G, Witjes JA, Compérat $E$ et al. EAU guidelines on primary urethral carcinoma. Eur Urol 2013; 64: 823-830.

4. Gheiler EL, Tefilli M, Tiguert R et al. Management of primary urethral cancer. Urology 1998; 52: 487-493

5. Dayyani $F$, Pettaway CA, Kamat AM et al. Retrospective analysis of survival outcomes and the role of cisplatin-based chemotherapy in patients with urethral carcinomas referred to medical oncologists. Urol Oncol 2013; 31: 1171-1177.

6. Carroll PR, Dixon CM. Surgical anatomy of the male and female urethra. Urol Clin North Am 1992; 19: 339-346.

7. Sobin LH, Wittekind C. TNM Classification of Malignant Tumors, 6th edition. New York: Wiley-Liss 2002.

8. Shim JW, Cho KS, Choi YD et al. Diagnostic algorithm for papillary urothelial tumors in the urinary bladder. Virchows Arch 2008; 452: 353-362.

9. Brunocilla $E$, Pernetti R, Martorana G. The prognostic role of Iymphovascular invasion in urothelial-cell carcinoma of upper and lower urinary tract. Anticancer Res 2011; 31: 3503-3506.

10. Rink M, Fajkovic H, Cha EK et al. Death certificates are valid for the determination of cause of death in patients with upper and lower tract urothelial carcinoma. Eur Urol 2012; 61: 854-855.

11. Gakis G, Efstathiou JA, Lerner SP et al. ICUD-EAU International Consultation on Bladder Cancer 2012: radical cystectomy and bladder preservation for muscleinvasive urothelial carcinoma of the bladder. Eur Urol 2013; 63: 45-57.

12. Kent M, Zinman L, Girshovich L, Sands J, Vanni A. Combined chemoradiation as primary treatment for invasive male urethral cancer. J Urol 2015; 193: 532-537.

13. Gakis G, Morgan TM, Efstathiou JA et al. Prognostic factors and outcomes in primary urethral cancer: results of the international collaboration on primary urethral carcinoma. World J Urol 2015 May 17 [epub ahead of print], doi: 10.1007/s00345015-1583-7. 